Case report

\title{
An unusual staining of the tooth roots: a case report with histological and micro-analytical studies
}

\author{
Yohko Fukuta, Yasunori Takeda ${ }^{\S}$, Yoshiyasu Fukuta ${ }^{\dagger}$, Morio Totsuka, Yasuo Yoshida $^{\ddagger}$, \\ Shyuichiro Hayashi ${ }^{\ddagger}$, Jiro Niitsu* and Hirotsugu Yamamoto
}

Departments of Oral Diagnosis, ${ }^{\S}$ Oral Pathology and ${ }^{\dagger}$ Oral and Maxillofacial Surgery, School of Dentistry, and

FElectron Microscope Laboratory, Iwate Medical University, Iwate 020-8505

*Dental Clinic of Morioka City Hospital, Iwate 020-0866

IDepartment of Pathology, Nihon University School of Dentistry at Matsudo, Chiba 271-8587

(Received 22 March and accepted 15 May 2001)

\begin{abstract}
The discoloration of tooth roots is rare. We report here a 22-year-old Japanese woman with blackish-brown staining of the roots of the upper and lower third molars. Staining was found in the dentin and cementum. Electron probe $\mathrm{X}$-ray microanalysis showed no significant difference in the composing elements between the stained tooth root and control tooth. Fluorescent bands coincided with staining in the dentin of the root and cementum along the incremental lines under confocal laser-scanning microscope. (J. Oral Sci. 43, 213-215, 2001)
\end{abstract}

Key words:staining; third molar; tooth root; dentin; cementum.

\section{Introduction}

There are various causes for the discoloration of teeth (1) such as surface deposits, changes in the structure or thickness of the dental hard tissues, diffusion of pigments into the dental hard tissues after formation, and the incorporation of pigments into the dental hard tissues during formation. When dental hard tissue is stained during the formation stage of the teeth, endogenous elements such as bile pigments and porphyrins, and exogenous ones such as tetracycline and heavy metals, are involved in the

Correspondence to Dr. Yohko Fukuta, Department of Oral Diagnosis, School of Dentistry, Iwate Medical University, 1-327 Chuo-dori, Morioka, Iwate 020-8508, Japan

Tel: +81-19-651-5111 ext. 4211

E-mail address: yfukukta@iwate-med.ac.jp staining. Discoloration of only the roots in the third molar is extremely rare.

We report a case of stained third molar roots, in which histological and analytical studies were performed using a stereomicroscope, an electron probe X-ray microanalyzer, and a confocal laser-scanning microscope.

\section{Case Report}

The patient was a 22-year-old Japanese woman with habitual cheek biting using the third molars. The patient had a history of slight neonatal jaundice, an allergic rash from 10 to 21 years of age, and acne for several years from the age of 12 years. Oral examination revealed that the left upper third molar was erupted and the lower bilateral third molars were partially erupted. These three molars were extracted.

\section{Observation of the Teeth Macroscopic findings \\ Extracted teeth showed marked blackish-brown-stained roots, but the crowns were normal in color (Fig. 1A). On the horizontally cut surfaces of the roots, the whole cementum and some areas of the dentin were dyed blackish brown (Fig. 1B).}

\section{Microscopic findings}

Half of the specimens were fixed in $10 \%$ neutral formalin, decalcified, and embedded in paraffin. The blackish-brown staining disappeared in this decalcified section.

The remaining half of the specimens were fixed in $10 \%$ neutral formalin and embedded in resin. On the horizontally cut surface, the cementum was dyed a deep 
blackish brown with a lamellar structure, and the dentin around the pulp chamber was brown (Fig. 2).

\section{Microfluorescent findings}

In the undecalcified ground section, examination using a microscope (Nikon E 1000: Nikon Ltd., Tokyo, Japan) with UV exciter a filter revealed fluorescence in the cervical portion of the dentin and around the pulp chamber in the root, and the whole cementum (Fig. 3A). Furthermore, using a confocal laser-scanning microscope (Olympus LSM-GB200: Olympus Ltd., Tokyo, Japan) under krypton-argon laser (488 nm, $568 \mathrm{~nm})$, strong fluorescence was noted along the incremental lines in the cervical portion of the dentin in the root and the whole cementum (Fig. 3B).

\section{Electron probe X-ray microanalysis}

The cut surfaces of the undecalcified sections were analyzed using an electron probe X-ray microanalyzer (JXA-8900L: JEOL Ltd., Tokyo, Japan). An upper third molar from a healthy 33-year-old woman was used as a control. There were no significant differences between the
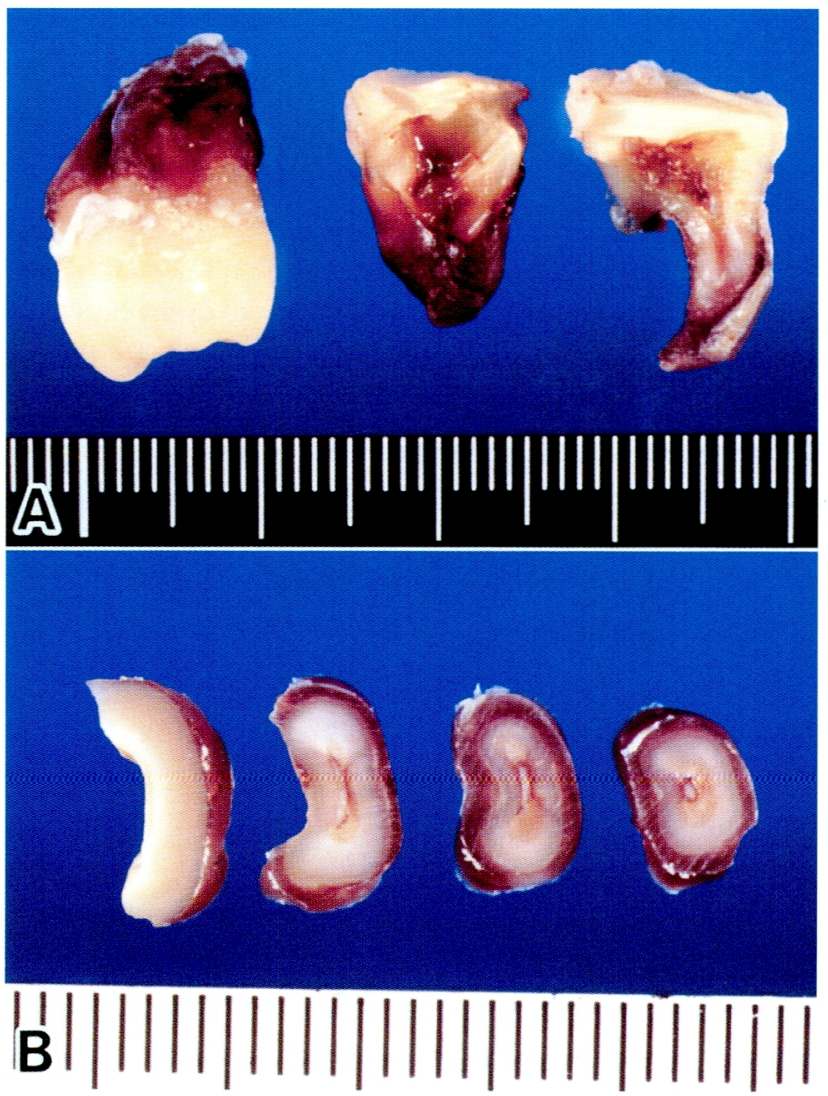

Fig. 1 Macroscopic view of the third molars showing deep blackish-brown staining of the roots.

A: Extracted teeth. B: Horizontally cut specimens of the tooth root. stained tooth roots and the control tooth in terms of the qualitative and quantitative distribution of the composing elements, such as $\mathrm{Ca}, \mathrm{P}, \mathrm{Na}, \mathrm{Mg}, \mathrm{Zn}, \mathrm{Fe}, \mathrm{Al}$ and $\mathrm{S}$.

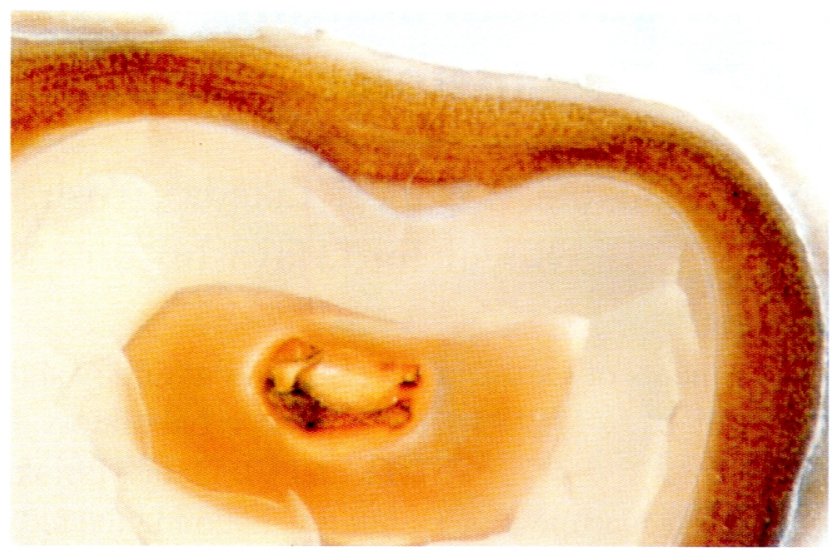

Fig. 2 Stereomicroscopic finding of the horizontally cut surface of the root embedded in resin. Deeply stained lamellar structure in the cementum $(\times 19)$.

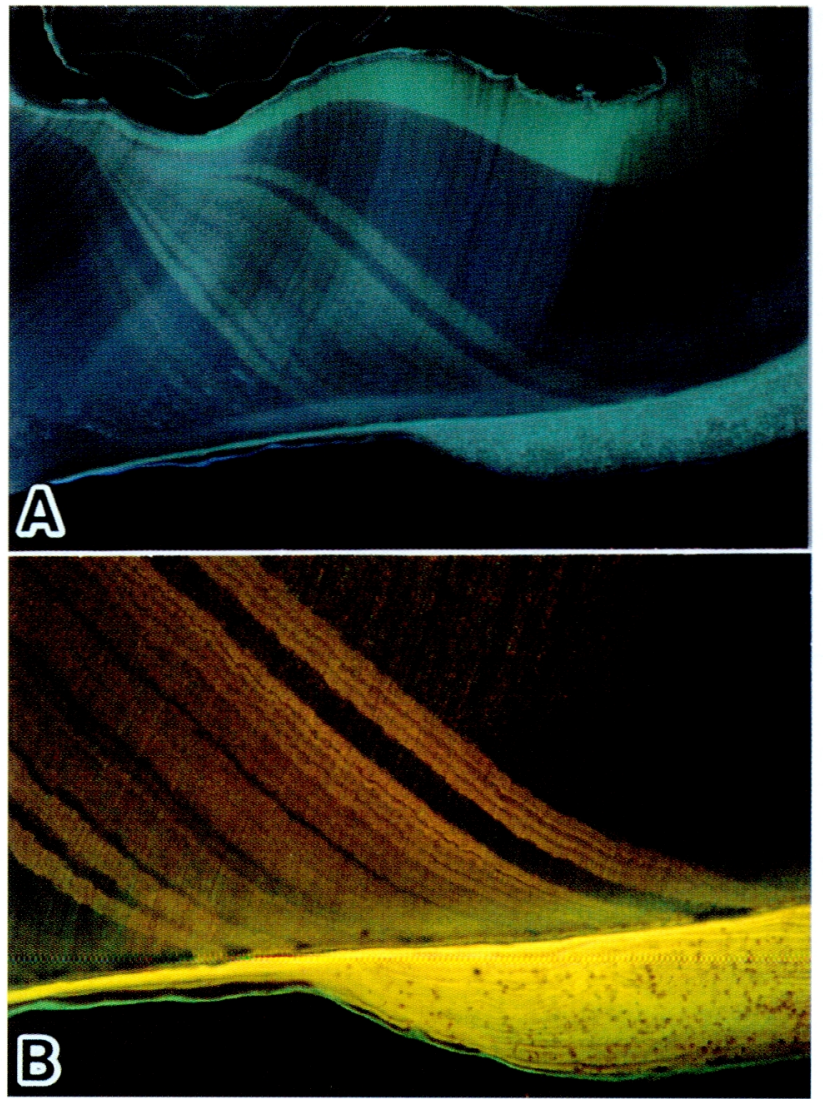

Fig. 3 Fluorescence of the tooth.

A: Fluorescence bands in the cervical portion of the dentin, around the pulp chamber of the dentin and cementum (Fluorescent microscopy, UV excitation, ground section, $\times 12)$.

B: Striped pattern along the incremental lines of the root dentin. Lamellar structure in the cellular cementum (Confocal laser-scanning microscopy, combination of $\mathrm{B}$ and $\mathrm{G}$ excitation, ground section, $\times 28$ ). 


\section{Discussion}

Discoloration, which is seen only in the tooth roots, is rare. However, in the present case, blackish-brown staining of the roots of the third molars was noted. In the third molar, crown formation is completed after age 12, and root formation is completed by 19 to 21 years of age (2). If something causes a discoloration effect during the root formation stage of the third molar, staining may only show in the root.

It is well known that various causes exist for staining of the tooth during formation of the tooth, such as hemolytic anemia (3), hyperbilirubinemia (4,5), congenital porphyria (6), and chronic renal failure (7). However, the patient here had no medical history of such diseases and no heavy metal was detected by an electron probe X-ray microanalyzer.

There have been a few reports of staining of the root of the third molars due to the use of tetracycline or minocycline for treating acne (8-10). Tetracycline is deposited in tooth as well as in bone, and minocycline, which is a semisynthetic derivative of tetracycline, is deposited in tooth, bone, skin, sclera, nail, and fatty tissues (9-13). Tetracycline can be identified by fluorescence with ultraviolet light with an absorption peak at 360 nanometers $(8,9)$, and fluorescent bands show under krypton-argon laser (488 $\mathrm{nm}$ ) using a confocal laser-scanning microscope (14). In the present case, the fluorescence was seen in the whole cementum, the cervical portion of the dentin and around the pulp chamber of the root. And, the patient had taken acne medicine for several years from age 12 years. We suspected that the stain was due to tetracycline administration during the root formation stage of these third molars. Tetracycline has a tendency to form a chelating compound with the formation of a tetracycline-calcium orthophosphate complex (12), and the disappearance of the staining in the decalcified section further supported our hypothesis.

\section{Acknowledgments}

This study was supported by Grants-in-Aid for High Performance Biomedical Materials Research from Ministry of Education, Culture, Sports, Science and Technology, Japan.

\section{References}

1. Soames, J. V. and Southam, J. C. (1998) Oral Pathology. 3rd ed., Oxford University Press, Oxford, 39-49

2. Moss-Salentijn, L. and Hendricks-Klyvert, M. (1990)
Dental and oral tissues. An Introduction. 3rd ed., Lea \& Febiger, Philadelphia, 165-203

3. Barta, J. E., King, D. L. and Jorgensen, R. L. (1989) $\mathrm{ABO}$ blood group incompatibility and primary tooth discoloration. Pediatr. Dent. 11, 316-318

4. Morisaki, I., Abe, K., Tong, L. S. M., Kato, K. and Sobue, S. (1990) Dental findings of children with biliary atresia: report of seven cases. ASDC J. Dent. Child. 57, 220-223

5. Watanabe, K., Shibata, T., Kurosawa, T., Morisaki, I., Kinehara, M., Igarashi, S. and Arisue, M. (1999) Bilirubin pigmentation of human teeth caused by hyperbilirubinemia. J. Oral Pathol. Med. 28, 128130

6. Fayle, S. A. and Pollard, M. A. (1994) Congenital erythropoietic porphyria.-oral manifestations and dental treatment in childhood: a case report. Quintessence Int. 25, 551-554

7. Wolff, A., Stark, H., Sarnat, H., Binderman, I., Eisenstein, B. and Drukker, A. (1985) The dental status of children with chronic renal failure. Int. J. Pediatr. Nephrol. 6, 127-132

8. Jackson, R. (1979) Tetracycline staining of wisdom teeth. Cutis 23, 613-616

9. Cohen, B. D. and Abrams, B. L. (1989) An unusual case of stained roots of unerupted third molars. Gen. Dent. 37, 342-343

10. Poliak, S. C., DiGiovanna, J. J., Gross, E. G., Gantt, G. and Peck, G.L. (1985) Minocycline-associated tooth discoloration in young adults. JAMA 254, 2930-2932

11. Wolfe, I. D. and Reichmister, J. (1984) Minocycline hyperpigmentation: skin, tooth, nail, and bone involvement. Cutis 33, 457-458

12. Sande, M. A. and Mandell, G. L. (1990) Antimicrobial agents. Tetracyclines, chloramphenicol, erythromycin, and miscellaneous antibacterial agents. In Goodman and Gilman's The pharmacological basis of therapeutics. 8th ed., Goodman Gilman, A., Rall, T.W., Nies, A. S. and Taylor, P. eds., Pergamon Press, New York, 11171145

13. Morrow, G. L. and Abbott, R. L. (1998) Minocycline-induced scleral, dental, and dermal pigmentation. Am. J. Ophthalmol. 125, 396-397

14. Love, R. M. and Chandler, N. P. (1996) A scanning electron and confocal laser microscope investigation of tetracycline-affected human dentine. Int. Endod. J. 29, 376-381 